\title{
ASYMPTOTICS OF THE PARTITION FUNCTION OF ISING MODEL ON INHOMOGENEOUS RANDOM GRAPHS
}

\author{
KWABEna Doku-Amponsah
}

\begin{abstract}
For a finite random graph, we defined a simple model of statistical mechanics. We obtain an annealed asymptotic result for the random partition function for this model on finite random graphs as $n$, the size of the graph is very large. To obtain this result, we define the empirical bond distribution, which enumerates the number of bonds between a given couple of spins, and empirical spin distribution, which enumerates the number of sites having a given spin on the spinned random graphs. For these empirical distributions we extend the large deviation principle(LDP) to cover random graphs with continuous colour laws. Applying Varandhan Lemma and this LDP to the Hamiltonian of the Ising model defined on Erdos-Renyi graphs, expressed as a function of the empirical distributions, we obtain our annealed asymptotic result.
\end{abstract}

Keywords: Large deviation principle, spinned graph, random partition function, free-energy density, empirical bond distribution, empirical spin distribution, Boltzmann distribution, Hamiltonian.

Mathematics Subject Classification : 46N55, 58Z05, 60F10,05C80.

\section{INTRODUCTION}

In this paper we study the partition function of the Ising model on an inhomogeneous random graph model defined as follows: we first divide vertices into two types, where the spin of every node is chosen independently and identically according to some spin law, then each bond is drawn independently with its probability given depending on the spins of the endpoints. This graph model is similar to the planted partition or stochastic block model, see [1, with the slight difference that in this model the edge probability within a block(type) is allowed to be different between the two blocks. On this random graph, we consider the Ising model and establish the asymptotic limit of the normalized log of the expected partition function, where the expectation is over the random graph.

The random partition function of random graphs has generated considerable interest in the mathematical physics community, see examples [2], [3],[4],[5],[6], [7], [8], [9], [10, [11], [12], [13], [14, for the understanding of the log partition function of constraint satisfaction problems(such as the Ising/Potts models, legal colourings, hardcore model etc.) on a random graph. See, [15], [16], [17], [18] for some recent applications of the Ising model on random graphs.

The most sought after quantity, which may be quite challenging to analyse in various models, is the quenched setting. The log-partition function is typically not concentrated and the analyses of the quenched log-partition is only possible for locally tree-like random graphs. [2], [8], [10, ,11, [12, [13. provide examples of the quenched asymptotic results for the log-partition function for the Ising model on general local tree-like shape random graphs which inhomogeneous Curie-Weiss and annealed random graphs. In [5], an annealed asymptotic result for the random partition function was first proved and the analogue of the quenched results were proved for the Potts model on a regular graph. 
The proofs in all these papers rely on the general locally homogeneous tree-like shape property of the random graphs; the configuration model, planted partition random graph model, stochastic block model. Some researchers, see e.g [14], have use the Monte Carlo simulations to investigate the asymptotic properties of the Ising model defined on Erdos-Renyi graphs.

In this paper we study statistical mechanic on random graphs which are locally non-homogeneous tree-like. Thus we study random graphs with a local structure of multitype Galton-Watson branching trees. To be specific, we use the large deviation principle (LDP) techniques developed in [19] and furthered in [20] to prove annealed asymptotic result for the log-partition functions of Ising model on inhomogeneous random graphs. Our annealed asymptotic result may serve as the basis for understanding the thermodynamic limiting behaviour of the free-energy function of the Ising model on inhomogeneous graphs.

The remaining part of the article is organized as follows: We present in Section 2 the Ising model on spinned graphs. The main results of this article, Theorem 3.1 and a Corollary [3.2 are stated in Section 3. Section 4 contains the LDP result and proof. Finally, we use our LDP results to prove our main Theorem and derive Corollary in Section 6.

\section{The Model}

By $\mathcal{V}=\{1,2,3, \ldots, n\}$ we denote by fixed set of $n$ sites and $E \subset \mathcal{E}:=\{(v, u) \in \mathcal{V} \times \mathcal{V}: v<u\}$, where the formal ordering of bonds is just to help us to simply describe unordered links. Let $\mathcal{X}=\{-1,+1\}$ be the spin set and define a function $\rho$ by $\rho: \mathcal{V} \rightarrow \mathcal{X}$.

For a symmetric function $q_{n}: \mathbb{R} \times \mathbb{R} \rightarrow[0,1]$, a continuous function $\sigma: \mathcal{X} \rightarrow \mathbb{R}$ and a probability distribution $\ell$ on $\sigma(\mathcal{X})=\{\sigma(-1), \sigma(+1)\}$ the Inhomogeneous spinned random graph or spinned graph $G$ with $n$ sites may be obtained as follows:

Each site $u \in \mathcal{V}$ with $\rho(u) \in \mathcal{X}$ is assigned magnetic ising spin $\sigma(\rho(u))$ independently according to the spin law $\ell$. Given the spins, we link any two sites $v, u \in \mathcal{V}$, independently according to a link probability $q_{n}[\sigma(\rho(v)), \sigma(\rho(u))]$ otherwise they remain disconnected.

We always observe $G=\{(\sigma(\rho(v)): v \in \mathcal{V}), E\}$ under the joint probability measure of graph and spin. We shall call $G$ spinned graph and observe $\sigma(\rho(v))$ as the spin of the site $v$.

On a spinned graph $X$, we define the ferromagnetic Ising Model by the following Boltzmann distributions over $\mathcal{X}^{\mathcal{V}}$,

$$
\mu_{G}(\rho)=\frac{1}{Z_{G}(\beta, B)} \exp \left\{\sum_{(v, u) \in E} \frac{\sigma(\rho(u))}{B(\rho(u))} \frac{\sigma(\rho(v))}{B(\rho(v))}+\sum_{u \in \mathcal{V}} \frac{\sigma(\rho(u))}{\sqrt{\beta}}\right\}
$$

where $\sigma(\rho(u))=\sqrt{\beta} B(\rho(u)) \rho(u), \beta \geq 0$ is the inverse temperature, $B=\left\{b(x): x \in\{-1,+1\}^{n}\right\}$ is the vector external of magnetic fields, $\rho(u) \in\{-1,+1\}$, and $Z_{G}$ the random partition function is given by

$$
Z_{G}(\beta, B):=\sum_{\rho \in\{-1,+1\}^{\mathcal{V}}} \exp \left\{\sum_{(v, u) \in E} \frac{\sigma(\rho(u))}{B(\rho(u))} \frac{\sigma(\rho(v))}{B(\rho(v))}+\sum_{u \in V} \frac{\sigma(\rho(u))}{\sqrt{\beta}}\right\} .
$$

Note $\frac{\sigma(\rho(v))}{B(\rho(v))}=0$ if $B(\rho(v))=0$ and also $\frac{\sigma(\rho(v))}{\sqrt{\beta}}=0$ if $\beta=0$.

Our main concern in this paper is the study of the annealed asymptotic of the partition function of the Ising model of spinned random graphs. We assume that $X$ is near-critical .i.e. the bond probabilities 
satisfy:

$$
\begin{aligned}
& \lim _{n \rightarrow \infty} n q_{n}[\sigma(+1), \sigma(+1)]=C[\sigma(+1), \sigma(+1)], \lim _{n \rightarrow \infty} n q_{n}[\sigma(-1), \sigma(+1)]=C[\sigma(-1), \sigma(+1)] \\
& \lim _{n \rightarrow \infty} n q_{n}[\sigma(+1), \sigma(-1)]=C[\sigma(+1), \sigma(-1)], \lim _{n \rightarrow \infty} n q_{n}[\sigma(-1), \sigma(-1)]=C[\sigma(-1), \sigma(-1)],
\end{aligned}
$$

where $C: \mathbb{R} \times \mathbb{R} \rightarrow[0, \infty)$ is a symmetric function and is not equal to zero identically. Throughout the rest part of the article we will write

$$
C_{i, j}=C[(\sigma(i), \sigma(j)]
$$

\section{Main Result}

We define $\lambda:[0,1] \rightarrow \mathbb{R}$ a function by

where

$$
\lambda(y)=a_{1} y^{2}+a_{2}(1-y)^{2}+a_{3} y(1-y)+B(1) y-B(-1)(1-y)
$$

$$
a_{1}(\beta, B)=\frac{1}{2} C_{+1,+1}\left(e^{\beta}-1\right), a_{2}(\beta, B)=\frac{1}{2} C_{-1,-1}\left(e^{\beta}-1\right) \text { and } a_{3}(\beta, B)=C_{-1,+1}\left(e^{-\beta}-1\right) .
$$

We recall that $B$ is a vector of magnetic field of the system and state the main result of this article, Theorem 3.1 .

Theorem 3.1. Suppose that $X$ is a spinned random graph with bond probabilities $q_{n}: \mathcal{X} \times \mathcal{X} \rightarrow[0,1]$ satisfying $n q_{n}[\sigma(e), \sigma(t)] \rightarrow C[\sigma(e), \sigma(t)]$, for all $t, e \in\{-1,+1\}$ and some continuous symmetric function $C: \mathbb{R} \times \mathbb{R} \rightarrow[0, \infty)$ with $\sup _{y, x \in \mathbb{R}} C[y, x]<\infty$. Let $Z_{G}(B, \beta)$ be random partition function of $X$. Then, $Z_{G}(B, \beta)$ satisfies

$$
\lim _{n \rightarrow \infty} \frac{1}{n} \log \mathbb{E}\left[Z_{G}(B, \beta)\right]=\sup _{y \in[0,1]}\{-y \log y-(1-y) \log (1-y)+\lambda(y)\} .
$$

From Theorem 3.1 we derive a corollary for the log- partition function of the Erdos-Renyi Graphs (where $n p_{n} \rightarrow c$ ) as a special case of random graphs with locally non-homogeneous tree-like property.

Corollary 3.2. Suppose that $X$ is an Erdos-Renyi random graph with bond probability such that $n q_{n} \rightarrow c$. Let $Z_{G}(B, \beta)$ be random partition function of $X$. Then, $Z_{G}(B, \beta)$ satisfies

$$
\begin{array}{r}
\lim _{n \rightarrow \infty} \frac{1}{n} \log \mathbb{E}\left[Z_{G}(B, \beta)\right]=\sup _{y \in[0,1]}\left\{-y \log y-(1-y) \log (1-y)+c\left(e^{\beta}-1\right)\left(y^{2}-y+\frac{1}{2}\right)\right. \\
\left.+c\left(e^{-\beta}-1\right)\left(y-y^{2}\right)+2 b y-b\right\},
\end{array}
$$

where $B(+1)=B(-1)=b$ is constant external magnetic field.

\section{LARGE DEVIATION PRINCIPLES FOR SPINNED RANDOM GRAPHS}

In this section, we review some large deviation results of [19], and extend the joint LDP for empirical distributions of coloured random graph to spinned random graphs.i.e. we assume a more general spin law $\ell: \mathbb{R} \rightarrow[0,1]$ with all its exponential moments finite and prove an LDP for this model in a topology generated by the total variation norm.

To begin, we recall some useful definitions and notations from [19]. A rate function is a non-constant, lower semi-continuous function $J$ from a polish space $\mathcal{L}$ into $[0, \infty]$, we called it good if the level sets $\{J(m) \leq \alpha\}$ are compact for every $\alpha \in[0, \infty)$. A functional $L$ from the set of finite spinned random 
graph model to $\mathcal{L}$ is said to satisfy a large deviation principle with rate function $J$ if, for all Borel sets $\Gamma \subset \mathcal{L}$,

$$
-\inf _{p \in \operatorname{int} \Omega} J(p) \leq \liminf _{n \rightarrow \infty} \frac{1}{n} \log P\{L(X) \in \Omega\} \leq \limsup _{n \rightarrow \infty} \frac{1}{n} \log P\{L(X) \in \Omega\} \leq-\inf _{p \in \operatorname{cl} \Omega} J(p),
$$

where $X$ under $P$ is a spinned random graph with $n$ sites and int $\Omega$ and cl $\Omega$ refer to the interior, resp. closure, of the set $\Omega$.

And, for any finite or countable set $\mathcal{Y}$ we denote by $\mathcal{L}(\mathcal{Y})$ the space of probability distributions, and by $\tilde{\mathcal{L}}(\mathcal{Y})$ the space of finite distributions on $\mathcal{Y}$, both endowed with the topology generated by the total variation norm. For $\omega \in \tilde{\mathcal{L}}(\mathcal{Y})$ we denote by $\|\omega\|$ its total mass. Further, if $\ell \in \tilde{\mathcal{L}}(\mathcal{Y})$ and $\nu \ll \ell$ we denote by

$$
H(\eta \| \ell)=\int_{\mathbb{R}} \eta[d y] \log \left(\frac{\eta[d y]}{\ell[d y]}\right)
$$

the relative entropy of $\eta$ with respect to $\ell$. We set $H(\eta \| \ell)=\infty$ if $\eta \nless \ell$. Finally, we denote by $\tilde{\mathcal{L}}_{*}(\mathcal{Y} \times \mathcal{Y})$ the subspace of symmetric distributions in $\tilde{\mathcal{L}}(\mathcal{Y} \times \mathcal{Y})$.

On each spinned graph $G=((\sigma(\rho(v)): u \in \mathcal{V}), E)$ with $n$ vertices, we define a probability distribution, the empirical spin distribution $\mathcal{M}_{G}^{1} \in \mathcal{L}(\mathcal{X})$, by

$$
\mathcal{M}_{G}^{1}[y]:=\frac{1}{n} \sum_{u \in \mathcal{V}} \delta_{\sigma(\rho(u))}(y), \quad \text { for } y \in \mathbb{R},
$$

and a symmetric finite distribution, the empirical bond distribution $\mathcal{M}_{G}^{2} \in \tilde{\mathcal{L}}_{*}(\mathbb{R} \times \mathbb{R})$, by

$$
\mathcal{M}_{G}^{2}[y, x]:=\frac{1}{n} \sum_{(v, u) \in E}\left[\delta_{(\sigma(\rho(v), \sigma(\rho(u))}+\delta_{(\sigma(\rho(u)), \sigma(\rho(v))}\right](y, x), \quad \text { for } x, y \in \mathbb{R} .
$$

The total mass $\left\|\mathcal{M}_{G}^{2}\right\|=2|E| / n$.

Theorem 4.1 (Extension). Suppose that $G$ is a spinned random graph with spin law $\ell$ such that $n^{-1} \log \ell(n) \rightarrow-\infty$ and bond probabilities $q_{n}: \mathbb{R} \times \mathbb{R} \rightarrow[0,1]$ satisfying $n q_{n}[d y, d x] \rightarrow C[d y, d x]$, for all $x, y \in \mathbb{R}$ and some bounded symmetric distribution $C: \mathbb{R} \times \mathbb{R} \rightarrow[0, \infty)$. Then, as $n \rightarrow \infty$, the pair $\left(\mathcal{M}_{G}^{1}, \mathcal{M}_{G}^{2}\right)$ satisfies a large deviation principle in $\mathcal{L}(\mathbb{R}) \times \tilde{\mathcal{L}}_{*}(\mathbb{R} \times \mathbb{R})$ with good rate function

$$
J(\eta, \varpi)=H(\eta \| \ell)+\frac{1}{2}[H(\omega \| C \eta \otimes \eta)+\|C \eta \otimes \eta\|-\|\omega\|] .
$$

where the distribution $C \eta \otimes \eta \in \tilde{\mathcal{L}}(\mathbb{R} \times \mathbb{R})$ is defined by $C \eta \otimes \eta[d y, d x]=C(x, y) \eta[d x] \eta[d y]$.

\section{Proof of Large Deviation Theorem Extension}

5.1 Exponential Change-of-Measure Given a bounded function $\tilde{f}: \mathcal{X} \rightarrow \mathbb{R}$ and a symmetric bounded function $\tilde{g}: \mathcal{X} \times \mathcal{X} \rightarrow \mathbb{R}$, we define the constant $U_{\tilde{f}}$ by

$$
U_{\tilde{f}}=\log \int_{\mathbb{R}} e^{\tilde{f}[x]} \ell[d x]
$$

and the function $\tilde{h}_{n}: \mathbb{R} \times \mathbb{R} \rightarrow \mathbb{R}$ by

$$
\tilde{h}_{n}[x, y]=\log \left[\left(1-q_{n}[d y, d x]+q_{n}[d y, d x] e^{\tilde{g}[x, y]}\right)^{-n}\right],
$$

for $a, b \in \mathcal{X}$. We use $\tilde{f}$ and $\tilde{g}$ to define (for very large $n$ ) a new spinned random graph as follows: 
- To the $n$ labelled sites in $\mathcal{V}$ we assign spins from $s(\mathcal{X})$ iid according to the spin law $\ell$ defined by

$$
\tilde{\ell}[d x]=e^{\tilde{f}[x]-U_{\tilde{f}} \ell[d x] .}
$$

- Given any two sites $v, u \in \mathcal{V}$, with $u$ carrying spin $x$ and $v$ carrying spin $y$, we link site $u$ to site $v$ with probability

$$
\tilde{q}_{n}[y, x]=\frac{q_{n}[d y, d x] e^{\tilde{g}[y, x]}}{\left(1-q_{n}[y, x]+q_{n}[d y, d x]\right) e^{\tilde{g}[y, x]}} .
$$

We denote the transformed law by $\tilde{P}$. We observe that $\tilde{\ell}$ is a probability distribution and that $\tilde{P}$ is absolutely continuous with respect to $P$ as, for any coloured graph $G=((\sigma(\rho(u)): u \in \mathcal{V}), E)$,

$$
\begin{aligned}
\frac{d \tilde{P}}{d P}(G) & =\prod_{u \in V} \frac{\tilde{\ell}[\sigma(\rho(u))]}{\ell[\sigma(\rho(u))]} \prod_{(v, u) \in E} \frac{\tilde{q}_{n}[\sigma(\rho(v)), \sigma(\rho(u))]}{q_{n}[\sigma(\rho(v)), \sigma(\rho(u))]} \prod_{(v, u) \notin E} \frac{1-\tilde{q}_{n}[\sigma(\rho(v)), \sigma(\rho(u))]}{1-q_{n}[\sigma(\rho(v)), \sigma(\rho(u))]} \\
& =\prod_{u \in V} \frac{\tilde{\ell}[\sigma(\rho(u))]}{\ell[\sigma(\rho(u))]} \prod_{(v, u) \in E} \frac{\tilde{q}_{n}[\sigma(\rho(v)), \sigma(\rho(u))]}{q_{n}[\sigma(\rho(v)), \sigma(\rho(u))]} \times \frac{n-n q_{n}[\sigma(\rho(v)), \sigma(\rho(u))]}{n-n \tilde{q}_{n}[\sigma(\rho(v)), \sigma(\rho(u))]} \prod_{(v, u) \in \mathcal{E}} \frac{n-n \tilde{q}_{n}[\sigma(\rho(v)), \sigma(\rho(u))]}{n-n q_{n}[\sigma(\rho(v)), \sigma(\rho(u))]} \\
& =\prod_{u \in V} e^{\tilde{f}[\sigma(\rho(u))]-U_{\tilde{f}}} \prod_{(v, u) \in E} e^{\tilde{g}[\sigma(\rho(v)), \sigma(\rho(u))]} \prod_{(v, u) \in \mathcal{E}} e^{\frac{1}{n} \tilde{h}_{n}[\sigma(\rho(v)), \sigma(\rho(u))]} \\
& =\exp \left(n\left\langle\mathcal{M}_{G}^{1}, \tilde{f}-U_{\tilde{f}}\right\rangle+n\left\langle\frac{1}{2} \mathcal{M}_{G}^{2}, \tilde{g}\right\rangle+n\left\langle\frac{1}{2} \mathcal{M}_{G}^{1} \otimes \mathcal{M}_{G}^{1}, \tilde{h}_{n}\right\rangle-\left\langle\frac{1}{2} \mathcal{M}_{\Delta}^{1}, \tilde{h}_{n}\right\rangle\right),
\end{aligned}
$$

where

$$
\mathcal{M}_{\Delta}^{1}=\frac{1}{n} \sum_{v \in V} \delta_{(\sigma(\rho(v)), \sigma(\rho(v)))}
$$

We write $\langle f, \eta\rangle:=\int_{\mathbb{R}} f[x] \eta[d x]$ and

$$
\langle g, \omega\rangle:=\int_{\mathbb{R} \times \mathbb{R}} g[y, x] \omega[d y, d x] .
$$

Lemma 5.1 (Euler's lemma). If $n q_{n}[d y, d x] \rightarrow C[d y, d x]$ for every $y, x \in \mathbb{R}$, then

$$
\lim _{n \rightarrow \infty}\left(1+\alpha q_{n}[y, x]\right)^{n}=e^{r C[d y, d x]}, \text { for all } y, x \in \mathbb{R} \text { and for } r \in \mathbb{R} \text {. }
$$

Proof. Notice that, for any $\varepsilon>0$ and for very large $n$ we have

$$
\left(1+\frac{r C[d y, d x]-\varepsilon}{n}\right)^{n} \leq\left(1+\alpha q_{n}[y, x]\right)^{n} \leq\left(1+\frac{r C[d y, d x]+\varepsilon}{n}\right)^{n},
$$

by the point-wise convergence. Hence by the sandwich theorem and Euler's formula we get (51).

Lemma 5.2 (Exponential tightness). For every $\Theta>0$ there exists $M \in \mathbb{N}$ such that

$$
\limsup _{n \rightarrow \infty} \frac{1}{n} \log P\{|E|>n M\} \leq-\Theta .
$$

Proof. Let $c>\sup _{x, y \in \mathbb{R}} C[d y, d x]>0$. Now, we use a coupling argument to define, for all large $n$, a new spinned random graph $\tilde{X}$ with spin law $\ell$ and bond probability $\frac{d}{n}$, such that any bond present 
in $X$ is also present in $\tilde{X}$. Let $|\tilde{E}|$ be the number of bonds of $\tilde{X}$. Then, by Chebyshev's inequality, the binomial distribution, and Lemma 5.1, we have that

$$
\begin{aligned}
P\{|\tilde{E}| \geq n w\} \leq e^{-n w} \mathbb{E}\left\{e^{|\tilde{E}|}\right\} & =e^{-n w} \sum_{k=0}^{\frac{n(n-1)}{2}} e^{k}\left(\begin{array}{c}
n(n-1) / 2 \\
k
\end{array}\right)\left(\frac{d}{n}\right)^{k}\left(1-\frac{d}{n}\right)^{n(n-1) / 2-k} \\
& =e^{-n l}\left(1-\frac{d}{n}+e \frac{d}{n}\right)^{n(n-1) / 2} \leq e^{-n w} e^{n d(e-1+o(1))} .
\end{aligned}
$$

Now given $\theta>0$ choose $M \in \mathbb{N}$ such that $M>\Theta+d(e-1)$ and observe that, for extremely large $n$,

$$
P\{|E| \geq n M\} \leq P\{|\tilde{E}| \geq n M\} \leq e^{-n \Theta},
$$

which implies the statement.

Lemma 5.3 (Exponential tightness). For every $\Theta>0$ there exists $K_{\Theta} \subset \mathcal{L}(\mathbb{R})$ such that

$$
\limsup _{n \rightarrow \infty} \frac{1}{n} \log P\left\{\mathcal{M}_{G}^{1} \notin K_{\Theta}\right\} \leq-\Theta .
$$

Proof. $\quad$ Let $w \in \mathbb{N}$ and choose $k(w) \in \mathbb{N}$, large enough, such that $\ell\left[e^{w^{2}} \mathbb{1}_{\{s>k(w)\}}\right] \leq 2^{w}$. Then, using exponential Chebyschev's inequality, we have that

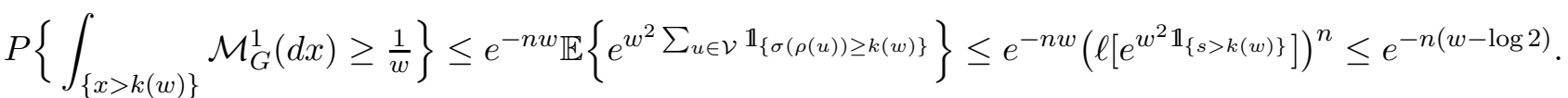

Now we fix $\Theta>0$, choose $M>\theta+\log 2$, define the set $\Gamma_{M}$ by

$$
\Gamma_{M}:=\left\{\eta: \int_{\{x \geq k(w)\}} \eta(d x)>\frac{1}{w}, \text { for all } w \geq M\right\}
$$

As $\{x \leq k(w)\} \subset \mathbb{R}$ is compact, the set $\Gamma_{M}$ is pre-compact in the weak topology, by Prohorov's criterion. As

we conclude that

$$
P\left\{\mathcal{M}_{G}^{1} \notin \Gamma_{M}\right\} \leq \frac{1}{1-e^{-1}} \exp (-n[M-\log 2]),
$$

$$
\limsup _{n \rightarrow \infty} \frac{1}{n} \log P\left\{\mathcal{M}_{G}^{1} \notin K_{\Theta}\right\} \leq-\Theta
$$

for the closure $K_{\Theta}$ of $\Gamma_{M}$ as required for the proof.

\subsection{Proof of the upper bound in Theorem 4.1}

Denote by $\mathcal{C}_{1}$ the space of bounded functions on $\mathbb{R}$ and by $\mathcal{C}_{2}$ the space of bounded symmetric functions on $\mathbb{R} \times \mathbb{R}$, and write

$$
\hat{J}(\eta, \varpi):=\sup _{\substack{f \in \mathcal{C}_{1} \\ g \in \mathcal{C}_{2}}}\left\{\int_{\mathbb{R}}\left(f[x]-U_{f}\right) \eta[d x]+\frac{1}{2} \int_{\mathbb{R} \times \mathbb{R}} g[y, x] \varpi[d y, d x]+\frac{1}{2} \int_{\mathbb{R} \times \mathbb{R}}\left(1-e^{g[y, x]}\right) C[d y, d x] \eta[d x] \eta[d y]\right\} .
$$

Lemma 5.4. For each closed set $F \subset \mathcal{L}(\mathbb{R}) \times \tilde{\mathcal{L}}_{*}(\mathbb{R} \times \mathbb{R})$,

$$
\limsup _{n \rightarrow \infty} \frac{1}{n} \log P\left\{\left(\mathcal{M}_{G}^{1}, \mathcal{M}_{G}^{2}\right) \in F\right\} \leq-\inf _{(\eta, \varpi) \in F} \hat{J}(\eta, \varpi) .
$$


Proof. First let $\tilde{f} \in \mathcal{C}_{1}$ and $\tilde{g} \in \mathcal{C}_{2}$ be arbitrary. Define $\tilde{\beta}: \mathbb{R} \times \mathbb{R} \rightarrow \mathbb{R}$ by

$$
\tilde{\beta}[y, x]=\left(1-e^{\tilde{g}[y, x]}\right) C[d y, d x] .
$$

Observe that, by Lemma [5.1, $\tilde{\beta}[y, x]=\lim _{n \rightarrow \infty} \tilde{h}_{n}[y, x]$ for all $a, b \in \mathbb{R}$, recalling the definition of $\tilde{h}_{n}$ from (3). Hence, by (4), for sufficiently large $n$,

$$
e^{\sup _{x \in \mathbb{R}}|\tilde{\beta}(x, x)|} \geq \int e^{\left\langle\frac{1}{2} \mathcal{M}_{\Delta}^{1}, \tilde{h}_{n}\right\rangle} d \tilde{P}=\mathbb{E}\left\{e^{n\left\langle\mathcal{M}_{G}^{1}, \tilde{f}-U_{\tilde{f}}\right\rangle+n\left\langle\frac{1}{2} \mathcal{M}_{G}^{2}, \tilde{g}\right\rangle+n\left\langle\frac{1}{2} \mathcal{M}_{G}^{1} \otimes \mathcal{M}_{G}^{1}, \tilde{h}_{n}\right\rangle}\right\},
$$

where $\mathcal{M}_{\Delta}^{1}=\frac{1}{n} \sum_{v \in \mathcal{V}} \delta_{(\sigma(\rho(v)), \sigma(\rho(v)))}$ and therefore,

$$
\limsup _{n \rightarrow \infty} \frac{1}{n} \log \mathbb{E}\left\{e^{n\left\langle\mathcal{M}_{G}^{1}, \tilde{f}-U_{\tilde{f}}\right\rangle+n\left\langle\frac{1}{2} \mathcal{M}_{G}^{2}, \tilde{g}\right\rangle+n\left\langle\frac{1}{2} \mathcal{M}_{G}^{1} \otimes \mathcal{M}_{G}^{1}, \tilde{h}_{n}\right\rangle}\right\} \leq 0 .
$$

Given $\varepsilon>0$ let $\hat{J}_{\varepsilon}(\eta, \varpi)=\min \left\{\hat{J}(\eta, \varpi), \varepsilon^{-1}\right\}-\varepsilon$. Suppose that $(\eta, \varpi) \in F$ and observe that $\hat{J}(\eta, \varpi)>$ $\hat{J}_{\varepsilon}(\eta, \varpi)$. We now fix $\tilde{f} \in \mathcal{C}_{1}$ and $\tilde{g} \in \mathcal{C}_{2}$ such that

$$
\left\langle\tilde{f}-U_{\tilde{f}}, \eta\right\rangle+\frac{1}{2}\langle\tilde{g}, \varpi\rangle+\frac{1}{2}\langle\tilde{\beta}, \eta \otimes \eta\rangle \geq \hat{J}_{\varepsilon}(\eta, \varpi) .
$$

As $\tilde{f}, \tilde{g}$ are bounded functions, there exist open neighbourhoods $B_{\varpi}^{2}$ and $B_{\eta}^{1}$ of $\varpi, \eta$ such that

$$
\inf _{\substack{\tilde{\tilde{n}} \in B_{\eta}^{1} \\ \tilde{\omega} \in B_{\varpi}^{2}}}\left\{\left\langle\tilde{f}-U_{\tilde{f}}, \tilde{\omega}\right\rangle+\frac{1}{2}\langle\tilde{g}, \tilde{\varpi}\rangle+\frac{1}{2}\langle\tilde{\beta}, \tilde{\omega} \otimes \tilde{\omega}\rangle\right\} \geq \hat{J}_{\varepsilon}(\eta, \varpi)-\varepsilon .
$$

Using Chebyshev's inequality and (6) we have that

$$
\begin{aligned}
\limsup _{n \rightarrow \infty} \frac{1}{n} & \log P\left\{\left(\mathcal{M}_{G}^{1}, \mathcal{M}_{G}^{2}\right) \in B_{\eta}^{1} \times B_{\varpi}^{2}\right\} \\
& \leq \limsup _{n \rightarrow \infty} \frac{1}{n} \log \mathbb{E}\left\{e^{n\left\langle\mathcal{M}_{G}^{1}, \tilde{f}-U_{\tilde{f}}\right\rangle+n\left\langle\frac{1}{2} \mathcal{M}_{G}^{2}, \tilde{g}\right\rangle+n\left\langle\frac{1}{2} \mathcal{M}_{G}^{1} \otimes \mathcal{M}_{G}^{1}, \tilde{h}_{n}\right\rangle}\right\}-\hat{J}_{\varepsilon}(\eta, \varpi)+\varepsilon \\
& \leq-\hat{J}_{\varepsilon}(\eta, \varpi)+\varepsilon .
\end{aligned}
$$

Now we use Lemma 5.2 and Lemma 5.3 with $\Theta=\varepsilon^{-1}$, to choose $M(\varepsilon) \in \mathbb{N}$ and $K_{\varepsilon}$ such that

$$
\limsup _{n \rightarrow \infty} \frac{1}{n} \log P\{|E|>n M(\varepsilon)\} \leq-\varepsilon^{-1} \text { and } \limsup _{n \rightarrow \infty} \frac{1}{n} \log P\left\{\mathcal{M}_{G}^{1} \notin K_{\varepsilon}\right\} \leq-\varepsilon^{-1}
$$

For this $M(\varepsilon)$ and $K_{\varepsilon}$ we define the set $\Gamma_{\varepsilon}$ by

$$
\Gamma_{\varepsilon}=\left\{(\eta, \varpi) \in \mathcal{L}(\mathbb{R}) \times \tilde{\mathcal{L}}_{*}(\mathbb{R} \times \mathbb{R}): \eta \in K_{\varepsilon}^{c},\|\varpi\| \leq 2 M(\varepsilon)\right\},
$$

and recall that $\left\|\mathcal{M}_{G}^{2}\right\|=2|E| / n$. The set $\Gamma_{\varepsilon} \cap F$ is compact and hence we can cover it by finitely many sets $B_{\eta_{r}}^{1} \times B_{\varpi_{r}}^{2}, r=1, \ldots, m$ with $\left(\eta_{r}, \varpi_{r}\right) \in F$ for $r=1, \ldots, m$. Consequently,

$$
P\left\{\left(\mathcal{M}_{G}^{1}, \mathcal{M}_{G}^{2}\right) \in F\right\} \leq \sum_{r=1}^{m} P\left\{\left(\mathcal{M}_{G}^{1}, \mathcal{M}_{G}^{2}\right) \in B_{\eta_{r}}^{1} \times B_{\varpi_{r}}^{2}\right\}+P\left\{\left(\mathcal{M}_{G}^{1}, \mathcal{M}_{G}^{2}\right) \notin \Gamma_{\varepsilon}\right\} .
$$

We may now use (7) and (8) to obtain, for all small $\varepsilon>0$,

$$
\begin{aligned}
\limsup _{n \rightarrow \infty} \frac{1}{n} \log P\left\{\left(\mathcal{M}_{G}^{1}, \mathcal{M}_{G}^{2}\right) \in F\right\} & \leq \max _{r=1}^{m}\left(\limsup _{n \rightarrow \infty} \frac{1}{n} \log P\left\{\left(\mathcal{M}_{G}^{1}, \mathcal{M}_{G}^{2}\right) \in B_{\eta_{r}}^{1} \times B_{\varpi_{r}}^{2}\right\}\right) \vee(-\varepsilon)^{-1} \\
& \leq\left(-\inf _{(\eta, \varpi) \in F} \hat{J}_{\varepsilon}(\eta, \varpi)+\varepsilon\right) \vee(-\varepsilon)^{-1} .
\end{aligned}
$$

Taking $\varepsilon \downarrow 0$ we get the desired statement. 
Moreover, we can write the rate function in terms of relative entropies, see [21, (2.15)], and hence we will show that the rate function is a good rate function. Recall the definition of the function $J$ from Theorem 4.1.

\section{Lemma 5.5.}

(i) $\hat{J}(\eta, \varpi)=J(\eta, \varpi)$, for any $(\eta, \varpi) \in \mathcal{L}(\mathbb{R}) \times \tilde{\mathcal{L}}_{*}(\mathbb{R} \times \mathbb{R})$,

(ii) $J$ is a good rate function and

(iii) $[H(\varpi \| C \eta \otimes \eta)+\|C \eta \otimes \eta\|-\|\varpi\|] \geq 0$ with equality iff $\varpi=C \eta \otimes \eta$.

Proof. (i) If $\varpi \nless C \eta \otimes \eta$, then, there exists $y_{0}, x_{0} \in \mathbb{R}$ with $C \eta \otimes \eta\left(y_{0}, x_{0}\right)=0$ and $\varpi\left(y_{0}, x_{0}\right)>0$. We define $\hat{g}: \mathbb{R} \times \mathbb{R} \rightarrow \mathbb{R}$ by

$$
\hat{g}[y, x]=\log \left[K\left(\mathbb{1}_{\left(y_{0}, x_{0}\right)}[y, x]+\mathbb{1}_{\left(y_{0}, x_{0}\right)}[y, x]\right)+1\right], \text { for } y, x \in \mathbb{R} \text { and } K>0 .
$$

This choice of $\hat{g}$ and $f=0$ gives

$$
\begin{aligned}
& \int_{\mathbb{R}}\left(f[x]-U_{f}\right) \eta(d x)+\int_{\mathbb{R} \times \mathbb{R}} \frac{1}{2} \hat{g}[y, x] \varpi[d y, d x]+\int_{\mathbb{R} \times \mathbb{R}} \frac{1}{2}\left(1-e^{\hat{g}[y, x]}\right) C[d y, d x] \eta[d x] \eta[d y] \\
& \geq \frac{1}{2} \log (K+1) \varpi\left(y_{0}, x_{0}\right) \rightarrow \infty, \quad \text { for } K \uparrow \infty .
\end{aligned}
$$

Now suppose that $\varpi \ll C \eta \otimes \eta$. We have

$$
\begin{aligned}
\hat{J}(\eta, \varpi)=\sup _{f \in \mathcal{C}_{1}}\left\{\int_{\mathbb{R}}\left(f[x]-\log \int_{\mathbb{R}} e^{f[x]} \ell[d x]\right) \eta[d x]\right\} \\
\quad+\frac{1}{2} \int_{\mathbb{R} \times \mathbb{R}} C[d y, d x] \eta[d x] \eta[d y]+\frac{1}{2} \sup _{g \in \mathcal{C}_{2}}\left\{\int_{\mathbb{R} \times \mathbb{R}} g[y, x] \varpi[d y, d x]-\int_{\mathbb{R} \times \mathbb{R}} e^{g[y, x]} C[d y, d x] \eta[d x] \eta[d y]\right\} .
\end{aligned}
$$

By the variational characterization of relative entropy, the first term equals $H(\eta \| \ell)$. By the substitution $h=e^{g} \frac{C \eta \otimes \eta}{\varpi}$ the last term equals

$$
\begin{aligned}
\sup _{\substack{h \in \mathcal{C}_{2} \\
h \geq 0}} & \int_{\mathbb{R} \times \mathbb{R}}\left[\log \left(h[y, x] \frac{\varpi[d y, d x]}{C[d y, d x] \eta[d x] \eta[d y]}\right)-h[y, x]\right] \varpi[d y, d x] \\
& =\sup _{\substack{h \in \mathcal{C}_{2} \\
h \geq 0}} \sum_{x, y \in \mathbb{R}}(\log h[y, x]-h[y, x]) \varpi[d y, d x]+\sum_{x, y \in \mathbb{R}} \log \left(\frac{\varpi[d y, d x]}{C[d y, d x] \eta[d x] \eta[d y]}\right) \varpi[d y, d x] \\
& =-\|\varpi\|+H(\varpi \| C \eta \otimes \eta),
\end{aligned}
$$

where we have used $\sup _{z>0} \log z-z=-1$ in the last line. This gives that $\hat{J}(\eta, \varpi)=J(\eta, \varpi)$.

(ii) We write $h_{C}(\varpi \| \eta):=[H(\varpi \| C \eta \otimes \eta)+\|C \eta \otimes \eta\|-\|\varpi\|]$ and notice that, all summands are continuous in $\eta, \varpi$. Thus $I$ is a rate function. Moreover, for all $r<\infty$, the level sets $\{J(\eta, \varpi) \leq r\}$ are contained in the bounded set $\left\{(\eta, \varpi) \in \mathcal{L}(\mathbb{R}) \times \tilde{\mathcal{L}}_{*}(\mathbb{R} \times \mathbb{R}): h_{C}(\varpi \| \eta) \leq r\right\}$ and are therefore compact. Consequently, $I$ is a good rate function.

(iii) We consider the non-negative function $\phi(z)=z \log z-z+1$, for $z>0, \phi(0)=1$, which has its only root in $z=1$. Note that

$$
h_{C}(\varpi \| \eta)= \begin{cases}\int \phi \circ g d C \eta \otimes \eta & \text { if } g:=\frac{d \varpi}{d C \eta \otimes \eta} \geq 0 \text { exists, } \\ \infty & \text { otherwise. }\end{cases}
$$

Hence $h_{C}(\varpi \| \eta) \geq 0$, and, if $\varpi=C \eta \otimes \eta$, then $\phi\left(\frac{d \varpi}{d C \eta \otimes \eta}\right)=\phi(1)=0$ and so $h_{C}(C \eta \otimes \eta \| \eta)=0$. Conversely, if $h_{C}(\varpi \| \eta)=0$, then $\varpi[d y, d x]>0$ implies $C \eta \otimes \eta[y, x]>0$, which then gives 
$\phi \circ g[y, x]=0$ and $g[y, x]=1$. Therefore, $\varpi=C \eta \otimes \eta$, which ends the proof of (iii) above.

\subsection{Proof of the lower bound in Theorem 4.1}

Lemma 5.6. For every open set $O \subset \mathcal{L}(\mathbb{R}) \times \tilde{\mathcal{L}}_{*}(\mathbb{R} \times \mathbb{R})$,

$$
\liminf _{n \rightarrow \infty} \frac{1}{n} \log P\left\{\left(\mathcal{M}_{G}^{1}, \mathcal{M}_{G}^{2}\right) \in O\right\} \geq-\inf _{(\eta, \varpi) \in O} J(\eta, \varpi) .
$$

Proof. $\quad$ Suppose we have $(\eta, \varpi) \in O$, with $\varpi \ll C \eta \otimes \eta$. Define $\tilde{f}_{\eta}: \mathbb{R} \rightarrow \mathbb{R}$ by

$$
\tilde{f}_{\eta}(a)=\left\{\begin{array}{ll}
\log \frac{\eta[d x]}{\ell[d x]}, & \text { if } \eta[d x]>0, \\
0, & \text { otherwise. }
\end{array}\right\} .
$$

and $\tilde{g}_{\varpi}: \mathbb{R} \times \mathbb{R} \rightarrow \mathbb{R}$ by

$$
\tilde{g}_{\varpi}[y, x]= \begin{cases}\log \frac{\varpi[d y, d x]}{C[d y, d x] \eta[d x] \eta[d y]}, & \text { if } \varpi[d y, d x]>0, \\ 0, & \text { otherwise. }\end{cases}
$$

In addition, we let $\tilde{\beta}_{\varpi}[y, x]=C[d y, d x]\left(1-e^{\tilde{g}_{\varpi}[y, x]}\right)$ and note that $\tilde{\beta}_{\varpi}[y, x]=\lim _{n \rightarrow \infty} \tilde{h}_{\varpi, n}[y, x]$, for all $x, y \in \mathbb{R}$ where

$$
\tilde{h}_{\varpi, n}[y, x]=\log \left[\left(1-q_{n}[d y, d x]+q_{n}[d y, d x] e^{\tilde{g}_{\varpi}[y, x]}\right)^{-n}\right] .
$$

Choose $B_{\eta}^{1}, B_{\varpi}^{2}$ open neighbourhoods of $\eta, \varpi$, such that $B_{\eta}^{1} \times B_{\varpi}^{2} \subset O$ and for all $(\tilde{\eta}, \tilde{\varpi}) \in B_{\eta}^{1} \times B_{\varpi}^{2}$

$$
\left\langle\tilde{f}_{\eta}, \eta\right\rangle+\frac{1}{2}\left\langle\tilde{g}_{\varpi}, \varpi\right\rangle+\frac{1}{2}\left\langle\tilde{\beta}_{\varpi}, \eta \otimes \eta\right\rangle-\varepsilon \leq\left\langle\tilde{f}_{\eta}, \tilde{\omega}\right\rangle+\frac{1}{2}\left\langle\tilde{g}_{\varpi}, \tilde{\varpi}\right\rangle+\frac{1}{2}\left\langle\tilde{\beta}_{\varpi}, \tilde{\omega} \otimes \tilde{\omega}\right\rangle .
$$

We now use $\tilde{P}$, the probability distribution obtained by transforming $P$ using the functions $\tilde{f}_{\eta}$, $\tilde{g}_{\varpi}$. Note that the spin law in the transformed distribution is now $\eta$, and the connection probabilities $\tilde{q}_{n}[d y, d x]$ satisfy $n \tilde{q}_{n}[d y, d x] \rightarrow(\varpi[d y, d x]) /(\eta[d x] \eta[d y])=: \tilde{C}[d y, d x]$, as $n \rightarrow \infty$. We use (44), to obtain

$$
\begin{aligned}
P\left\{\left(\mathcal{M}_{G}^{1}, \mathcal{M}_{G}^{2}\right)\right. & \in O\} \geq \tilde{\mathbb{E}}\left\{\frac{d P}{d \tilde{P}}(G) \mathbb{1}_{\left\{\left(\mathcal{M}_{G}^{1}, \mathcal{M}_{G}^{2}\right) \in B_{\eta}^{1} \times B_{\varpi}^{2}\right\}}\right\} \\
& =\tilde{\mathbb{E}}\left\{\prod_{u \in V} e^{-\tilde{f}_{\eta}[\sigma(\rho(u))]} \prod_{(v, u) \in E} e^{-\tilde{g}_{\varpi}[\sigma(\rho(v)), \sigma(\rho(u))]} \prod_{(v, u) \in \mathcal{E}} e^{-\frac{1}{n} \tilde{h}_{\varpi, n}[\sigma(\rho(v)), \sigma(\rho(u))]} \mathbb{1}_{\left\{\left(\mathcal{M}_{G}^{1}, \mathcal{M}_{G}^{2}\right) \in B_{\eta}^{1} \times B_{\varpi}^{2}\right\}}\right\} \\
& =\tilde{\mathbb{E}}\left\{e^{-n\left\langle L^{1}, \tilde{f}_{\eta}\right\rangle-n \frac{1}{2}\left\langle L^{2}, \tilde{g}_{\varpi}\right\rangle-n \frac{1}{2}\left\langle\mathcal{M}_{G}^{1} \otimes \mathcal{M}_{G}^{2}, \tilde{g}_{\varpi}\right\rangle+\frac{1}{2}\left\langle\mathcal{M}_{\Delta}^{1}, \tilde{h}_{\varpi, n}\right\rangle} \times \mathbb{1}_{\left\{\left(\mathcal{M}_{G}^{1}, \mathcal{M}_{G}^{2}\right) \in B_{\eta}^{1} \times B_{\varpi}^{2}\right\}}\right\} \\
& \geq \exp \left(-n\left\langle\tilde{f}_{\eta}, \eta\right\rangle-n \frac{1}{2}\left\langle\tilde{g}_{\varpi}, \varpi\right\rangle-n \frac{1}{2}\left\langle\tilde{\beta}_{\varpi}, \eta \otimes \eta\right\rangle+m-n \varepsilon\right) \times \tilde{P}\left\{\left(\mathcal{M}_{G}^{1}, \mathcal{M}_{G}^{2}\right) \in B_{\eta}^{1} \times B_{\varpi}^{2}\right\}
\end{aligned}
$$

where $m:=0 \wedge \inf _{x \in \mathbb{R}} \tilde{\beta}[x, x]$. Therefore, by (5), we have

$$
\begin{aligned}
& \liminf _{n \rightarrow \infty} \frac{1}{n} \log P\left\{\left(\mathcal{M}_{G}^{1}, \mathcal{M}_{G}^{2}\right) \in O\right\} \\
& \geq-\left\langle\tilde{f}_{\eta}, \eta\right\rangle-\frac{1}{2}\left\langle\tilde{g}_{\varpi}, \varpi\right\rangle-\frac{1}{2}\left\langle\tilde{\beta}_{\varpi}, \eta \otimes \eta\right\rangle-\varepsilon+\liminf _{n \rightarrow \infty} \frac{1}{n} \log \tilde{P}\left\{\left(\mathcal{M}_{G}^{1}, \mathcal{M}_{G}^{2}\right) \in B_{\eta}^{1} \times B_{\varpi}^{2}\right\} .
\end{aligned}
$$

The result follows once we prove that

$$
\liminf _{n \rightarrow \infty} \frac{1}{n} \log \tilde{P}\left\{\left(\mathcal{M}_{G}^{1}, \mathcal{M}_{G}^{2}\right) \in B_{\eta}^{1} \times B_{\varpi}^{2}\right\}=0 .
$$


We apply the upper bound (but now with $\tilde{P}$ in place of $P$ ) to prove (11). Then we obtain

$$
\limsup _{n \rightarrow \infty} \frac{1}{n} \log \tilde{P}\left\{\left(\mathcal{M}_{G}^{1}, \mathcal{M}_{G}^{2}\right) \in\left(B_{\eta}^{1} \times B_{\varpi}^{2}\right)^{c}\right\} \leq-\inf _{(\tilde{\eta}, \tilde{\varpi}) \in \tilde{F}} \tilde{J}(\tilde{\eta}, \tilde{\varpi})
$$

where $\tilde{F}=\left(B_{\eta}^{1} \times B_{\varpi}^{2}\right)^{c}$ and $\tilde{J}(\tilde{\eta}, \tilde{\varpi}):=H(\tilde{\eta} \| \eta)+\frac{1}{2} h_{\tilde{C}}(\tilde{\varpi} \| \tilde{\eta})$. It therefore reduces to showing that the infimum is non-negative. Suppose that by contradiction we have a sequence $\left(\tilde{\eta}_{n}, \tilde{\varpi}_{n}\right) \in \tilde{F}$ with $\tilde{J}\left(\tilde{\eta}_{n}, \tilde{\varpi}_{n}\right) \downarrow 0$. Then, as $\tilde{J}$ is a good rate function and its level sets are compact, and by lower semi-continuity of the map $(\tilde{\eta}, \tilde{\varpi}) \mapsto \tilde{J}(\tilde{\eta}, \tilde{\varpi})$, we can find a limit point $(\tilde{\eta}, \tilde{\varpi}) \in \tilde{F}$ with $\tilde{J}(\tilde{\eta}, \tilde{\varpi})=0$ . By Lemma 5.5 this implies $H(\tilde{\eta} \| \eta)=0$ and $h_{C}(\tilde{\varpi} \| \tilde{\eta})=0$, hence $\tilde{\eta}=\eta$, and $\tilde{\varpi}=\tilde{C} \tilde{\eta} \otimes \tilde{\eta}=\varpi$ contradicting $(\tilde{\eta}, \tilde{\varpi}) \in \tilde{F}$.

\section{Proof of Main Results}

6.1 Proof of Theorem 3.1 We kick start the proof of our Thermodynamics limit results by stating an important Lemma (Varadhan's Lemma, see [21, Theorem 4.3.1] ), which is a key step in establishing our first result Theorem 3.1, without proof.

Lemma 6.1 (Varadhan). Suppose the functional $M_{n}$ from the space of finite spinned graphs to $\mathcal{L}$ satisfies the $L D P$ withh good rate function $J: \mathcal{L} \rightarrow[0, \infty]$ and let $\Psi: \mathcal{L} \rightarrow \mathbb{R}$ be any continuous function. Assume further the following moment condition for some $\lambda>1$,

$$
\limsup _{n \rightarrow \infty} \frac{1}{n} \log \mathbb{E}\left[e^{n \lambda \Psi\left(M_{n}[X]\right)}\right]<\infty .
$$

Then,

$$
\lim _{n \rightarrow \infty} \frac{1}{n} \log \mathbb{E}\left[e^{n \lambda \Psi\left(M_{n}[X]\right)}\right]=\sup _{m \in \mathcal{L}}\{\Psi(m)-J(m)\} .
$$

Now we prove an annealed asymptotic result for the partition function for the ferromagnetic Ising model on spinned random graphs, as the graph size goes to infinity.

Proof. Recall the partition function of the Ising model on the spinned random graph network from section 2 and write it as integration of some function with respect to our empirical distributions:

$$
\mathrm{E}\left[Z_{G}(B, \beta)\right]:=2^{n} \mathbb{E}\left[\exp \left\{\frac{n}{2} \int \frac{\sigma(x)}{B(x)} \frac{\sigma(x)}{B(y)} \mathcal{M}_{G}^{2}[d \sigma(x), d \sigma(y)]+n \int \frac{\sigma(x)}{\sqrt{\beta}} \mathcal{M}_{G}^{1}[d \sigma(x)]\right\}\right]
$$

Now using the (Varadhan) Lemma 6.1 and Theorem 4.1 we obtain,

$$
\begin{aligned}
& \lim _{n \rightarrow \infty} \frac{1}{n} \log \mathrm{E}\left[Z_{G}(B, \beta)\right]=\log 2+ \sup \left\{\frac{1}{2} \int \frac{\sigma(x)}{B(x)} \frac{\sigma(x)}{B(y)} \varpi[d \sigma(x), d \sigma(y)]+\int \frac{\sigma(x)}{\sqrt{\beta}} \eta[d \sigma(x)]-J(\eta, \varpi)\right. \\
&\left.: \eta \in \mathcal{X}(\{\sigma(-1), \sigma(+1)\}), \varpi \in \mathcal{L}_{*}(\{\sigma(-1), \sigma(+1)\} \times\{\sigma(-1), \sigma(+1)\})\right\} \\
&=\sup \left\{\frac{\beta}{2}\left(\varpi(\Delta)-\varpi\left(\Delta^{\mathrm{c}}\right)\right)+B(1) t-B(-1)(1-t)-t \log (t)-(1-t) \log (1-t)\right. \\
&\left.-\frac{1}{2}\left(H\left(\varpi \| \eta_{t}\right)+C_{+1,+1} t+C_{-1,-1}(1-t)+2 C_{-1,+1} t(1-t)-\|\varpi\|\right)\right\},
\end{aligned}
$$

where $\Delta$ is the diagonal in $\{\sigma(-1), \sigma(+1)\} \times\{\sigma(-1), \sigma(+1)\}$, and the supremum is over all $t \in$ $[0,1]$ and $\varpi \in \mathcal{L}_{*}(\{\sigma(-1), \sigma(+1)\} \times\{\sigma(-1), \sigma(+1)\})$, and the distribution $\eta_{t} \in \tilde{\mathcal{L}}_{*}(\{\sigma(-1), \sigma(+1)\} \times$ $\{\sigma(-1), \sigma(+1)\})$ is defined by

$$
\eta_{t}[s(i), s(j)]=C_{i, j} t^{(2+i+j) / 2}(1-t)^{(2-i-j) / 2} \text { for } i, j \in\{-1,+1\} .
$$


We take the partial derivatives of (14) with respect to $\varpi$, and set our results to zero to get,

$$
\begin{gathered}
\varpi[\sigma(+1), \sigma(+1)]=e^{\beta} \eta_{t}[\sigma(+1), \sigma(+1)], \\
\varpi[\sigma(-1), \sigma(-1)]=e^{\beta} \eta_{t}[\sigma(-1), \sigma(-1)] \\
\varpi[\sigma(+1), \sigma(-1)]=e^{-\beta} \eta_{t}[\sigma(+1), \sigma(-1)] \\
\varpi[\sigma(-1), \sigma(+1)]=e^{-\beta} \eta_{t}[\sigma(-1), \sigma(+1)] .
\end{gathered}
$$

Therefore writing $\varpi$ in (14) we obtain

$$
\begin{aligned}
& \lim _{n \rightarrow \infty} \frac{1}{n} \log \mathrm{E}\left[Z_{G}(B, \beta)\right] \\
& =\sup _{t \in[0,1]}\left\{\frac{\beta}{2} e^{\beta} \eta_{t}[\sigma(+1), \sigma(+1)]+\frac{\beta}{2} e^{\beta} \eta_{t}[\sigma(-1), \sigma(-1)]-\beta e^{-\beta} \eta_{t}[\sigma(-1), \sigma(+1)]+B(1) x-B(-1)(1-t)\right. \\
& -t \log (t)-(1-t) \log (1-t)-\frac{\beta}{2} e^{\beta} \eta_{t}[\sigma(+1), \sigma(+1)]-\frac{\beta}{2} e^{\beta} \eta_{t}[\sigma(-1), \sigma(-1)]+\beta e^{-\beta} \eta_{t}[\sigma(-1), \sigma(+1)]-\frac{1}{2} C_{+1,+1} t \\
& \left.-\frac{1}{2} C_{-1,-1}(1-t)-C_{-1,+1} t(1-t)+\frac{1}{2} e^{\beta} \eta_{t}[\sigma(+1), \sigma(+1)]+\frac{1}{2} e^{\beta} \eta_{t}[\sigma(-1), \sigma(-1)]+e^{-\beta} \eta_{t}[\sigma(-1), \sigma(+1)]\right\} \\
& =\sup _{t \in[0,1]}\left\{-t \log (t)-(1-t) \log (1-t)-\frac{1}{2} C_{+1,+1} t^{2}-\frac{1}{2} C_{-1,-1}(1-t)^{2}+B(1) x-B(-1)(1-t)\right. \\
& =\sup _{t \in[0,1]}\left\{-t \log (t)-(1-t) \log (1-t)+\frac{1}{2} C_{+1,+1}\left(e^{\beta}-1\right) x^{2}+\frac{1}{2} C_{-1,-1}\left(e^{\beta}-1\right)(1-t)^{2}+B(1) t-B(-1)(1-t)+C\right. \\
& =\sup _{t \in[0,1]}\left\{-t \log t-(1-t) \log (1-t)+a_{1} t^{2}+a_{2}(1-t)^{2}+a_{3} t(1-t)+B(1) t-B(-1)(1-t)\right\} \\
& =\sup _{t \in[0,1]}\{-t \log t-(1-t) \log (1-t)+\lambda(t)\}
\end{aligned}
$$

which ends the proof of our main Theorem.

6.2 Proof of Corollary 3.2 . Note that in case of the Erdos-Renyi graphs $C_{i, j}=c$ for all $i, j=-1,1$, with $n p_{n} \rightarrow c$ and $B(1)=B(-1)=b$. Therefore we can invoke Theorem 3.1 to obtain (11) as required. This ends the proof.

\section{Summary, Discussions and Future Work}

In this article we have found annealed asymptotic result for the random partition function of the Ising model on inhomogeneous graphs. The main technique used to establish the result is joint LDP for suitably defined empirical measures of the spinned random graph. Thus, we defined for each spinned random graph an empirical spin measure and empirical bond measure. For these empirical measures, the joint LDP of [17] is extended to cover continuous spins space. Note that the hamiltonian of the Ising model was then written as the integral of a function and the empirical measures. The Varadhan's Lemma, see [21, Theorem 4.3.1] is applied to the LDP to establish the annealed asymptotic result for normalized log expected value of the random partition function and the main result was obtained as a solution of the optimization problem 14. Elementary calculus gave us the solution of 14 which ends the proof of the main results. 
Corollary 3.2 above is qualitatively different from [22, Theorem 5.1] in two folds: First the result in this paper is an annealed results for the log-partition of the Erdos-Renyi graphs while [22, Theorem 5.1] gives a quenched result for the log-partition function. Second, finding explicit value for the normalized log-partition in [22, Theorem 5.1] will involve the computation of expectations with respect to the law of a local tree and might be very difficult without a numerical integration. However, Corollary 3.2 of this paper presents the limit of the normalized log- expected partition function as a simple closed optimization problem.

The same method may be adapted for random graphs mentioned in the Thesis of Dommers [22, Page 30] but not studied as their local structure is not tree-like. i.e conguration model with a household structure and scale-free percolation clusters and the fitness preferential attachment model. Combinatoric arguments through the method of types similar to the one deployed in the paper [19] will give the LDP.

\section{REFERENCES}

[1] T. A. B. Snijders and K. Nowicki. Estimation and Prediction for Stochastic Blockmodels for Graphs with Latent Block Structure. Journal of Classication 14 (1997), no. 1, 75100 .

[2] G. BARA and E. AgLiARI. Equilibrium statistical mechanics on correlated random graphs. J.Stat. Mech. (2011) P02027.

[3] A. Bovier. Statistical mechanics of disordered systems: a mathematical perspective. Cambridge University Press, 2006. Cambridge University Press, 2006.

[4] A. Dembo, A. Montanari and N. Sun. Factor models on locally tree-like graphs. The annals of Applied Probability, 41(6),pp.4162-4213,(2013)

[5] A. Dembo, A. Montanari, A. Sly and N. Sun The replica symmetric solution for Potts models on d-regular graphs. Comm. Math. Phy. 327:2(2014),pp. 551-575.

[6] A. Dembo and A. Montanari. Ising models on locally tree-like graphs. The annals of Applied Probability, 20(2):565-592,(2010)

[7] S. Dommers, C. Giardina. and R.V.D. Hofstad Ising models on power-law radom graphs. J Stat Phys (2010) 141: 638660 DOI 10.1007/s10955-010-0067-9 p

[8] S. N. Dorogovtsev,A. V. Goltsev and J.F.F. Mendes. Ising model on networks with an arbitrary distribution of connections Physical Review E, 66(1):1-5(2002).

[9] S. Dommers ,C. Giardina, C. Giberti and R. van der Hofstad Ising critical exponents on random trees and graphs. Comm. in Math. Physics, 328(1):335-395(2014).

[10] C. Giardina, C. Giberti, R. van der Hofstad and M.L. Prioriello. Quenched central limit theorems for the Ising Model on random graphs. J.Stat. Physics, 160(6):16231657(2015).

[11] C. Giardina, C. Giberti, R. van der Hofstad and M.L. Prioriello. Ising critical behaviour of inhomogeneous Curie-Weiss and annealed random graphs. arxiv:1509.0732.

[12] L. De SAnctis and F. Guerra. Mean field dilute ferromagnet:High temperature and zero temperature behaviour. J.Stat. Physics, 132(5):759-785(2008).

[13] Y. Deshrande,E. Abbe and A. Montanari. Asymptotic mutual information for the two-groups stochastic block model. arXiv:150\%.08685 
[14] E. Agliari,R. Burioni and P. Sgrignoli A Two-Population Ising model on diluted random graphs Preprint, arXiv: 1009.0251v1[cond-mat.sat-mech] 1 Sep 2010.

[15] E. Agliari and A. BARRA. A statistical mechanics approach to granovetter theory. Physica A 319, 3017-3026(2011).

[16] A. Dibiaso, E. Agliari, A. Barra and R. Burioni. Mean-field cooperativity in chemical kinetics. Theo. Chemistry Accounts, 131:1104(2012).

[17] G. Tkacik, C. Schneidman, M.J. Berry II, and W. Bialek. Ising models for networks of real neurons. Arxiv:q-bio/0611072,2006.

[18] J. Xie, J. Emenheiser, M. Kirby, S. Sreenivasan, B.K. Szymanski and G. KoRNISS. Evolution of Opinions on Social Networks in Presence of Competing Committed Groups. PloS One 7(3):e33215.(2012)

[19] K. Doku-Amponsah and P. Mörters. Large deviation principle for empirical measures of coloured random graphs. Ann. Appl. Prob. Volume 20, Number 6 (2010), 1989-2021.

[20] K. Doku-Amponsah. Asymptotic equipartition properties for hierarchical and networked structures. ESAIM: Probability and Statistics.DOI: 10.1051/ps/2010016 : Published online by Cambridge University Press: 03 February 2011.

[21] A. Dembo and O. Zeitouni. Large deviations techniques and applications. Springer, New York, (1998).

[22] S. Dommers. Spin models on random graphs. PhD Thesis, Eindhoven(2013).

$$
\text { University of Ghana }
$$

Statistics Department, Box LG 115, Legon, Ghana

Email: kdoku-amponsah@ug.edu.gh 